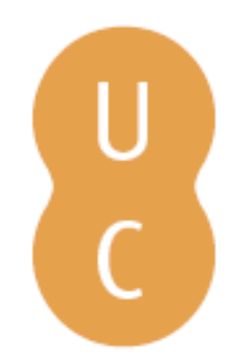

\title{
nombalina
}

\section{Einstein em Portugal: o primeiro teste da teoria da relatividade geral e o seu impacto na comunidade científica nacional}

\author{
Autor(es): $\quad$ Mota, Elsa; Simões, Ana; Crawford, Paulo \\ Publicado por: Imprensa da Universidade de Coimbra \\ URL \\ persistente: URI:http://hdl.handle.net/10316.2/32495 \\ DOI: $\quad$ DOI:http://dx.doi.org/10.14195/978-989-26-0353-7_4 \\ Accessed : $\quad$ 26-Apr-2023 07:48:20
}

A navegação consulta e descarregamento dos títulos inseridos nas Bibliotecas Digitais UC Digitalis, UC Pombalina e UC Impactum, pressupõem a aceitação plena e sem reservas dos Termos e Condições de Uso destas Bibliotecas Digitais, disponíveis em https://digitalis.uc.pt/pt-pt/termos.

Conforme exposto nos referidos Termos e Condições de Uso, o descarregamento de títulos de acesso restrito requer uma licença válida de autorização devendo o utilizador aceder ao(s) documento(s) a partir de um endereço de IP da instituição detentora da supramencionada licença.

Ao utilizador é apenas permitido o descarregamento para uso pessoal, pelo que o emprego do(s) título(s) descarregado(s) para outro fim, designadamente comercial, carece de autorização do respetivo autor ou editor da obra.

Na medida em que todas as obras da UC Digitalis se encontram protegidas pelo Código do Direito de Autor e Direitos Conexos e demais legislação aplicável, toda a cópia, parcial ou total, deste documento, nos casos em que é legalmente admitida, deverá conter ou fazer-se acompanhar por este aviso.

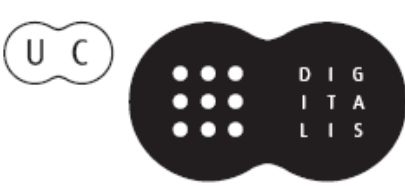




\section{CARLOS FIOLHAIS}

Coordenaç̃o

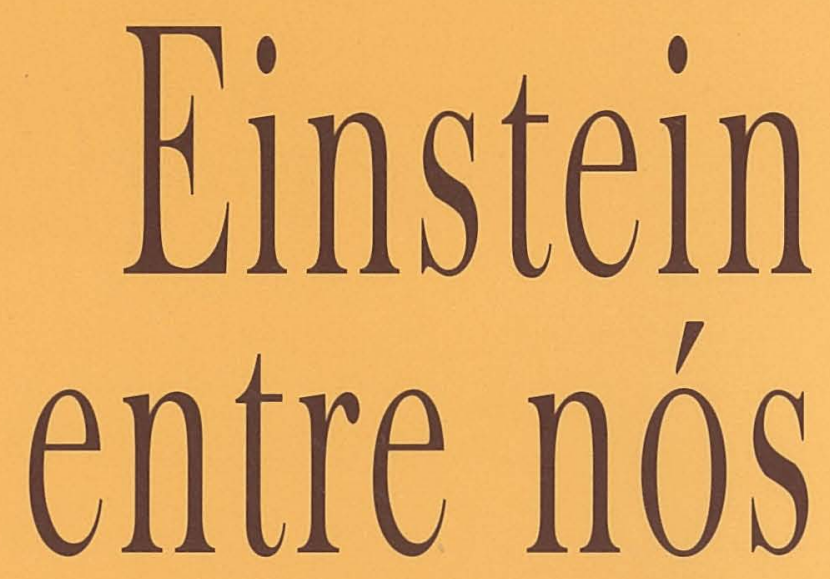

A recepcão de Einstein em Portugal

de 1905 a 1955

Coimbra Imprensa da Universidade $\quad 2005$ 
(Página deixada propositadamente em branco) 


\section{CARLOS FIOLHAIS}

Coordenação

\section{Einstein entre nós}

A recepscão de Einstein em Portugal

de 1905 a 1955

Coimbra I I prensa da Universidade $\quad 2005$ 


\section{Coordenação editorial}

Imprensa da Universidade de Coimbra

\section{Concepção gráfica}

António Barros

\section{Digitalização de imagens}

Alexandre Ramires

Museu de Ciência da Universidade de Lisboa

Paginação

SerSilito

\section{Execução gráfica \\ SerSilito - Maia}

\section{ISBN}

972-8704-60-7

\section{Depósito legal \\ 233349/05}

Outubro de 2005

(C) 2005, Imprensa da Universidade de Coimbra

\section{Apoios:}

Departamento de Física da Faculdade de Ciências e

Tecnologia da Universidade de Coimbra

Sociedade Portuguesa de Física

Associação para o Desenvolvimento do Departamento de Física (ADDF)

Programa Operacional Ciência e Inovação 2010

Museu de Ciência da Universidade de Lisboa 


\section{Einstein em Portugal: \\ O PRIMEIRO TESTE DA TEORIA DA RELATIVIDADE GERAL \\ E O SEU IMPACTO NA COMUNIDADE CIENTÍFICA NACIONAL}

Elsa Mota ${ }^{(*)}$; Ana Simões $\left({ }^{*}\right)$; Paulo Crawford $\left({ }^{* *}\right)$

\section{Introdução}

Em Novembro de 1915, após vários anos de tentativas infrutíferas, Albert Einstein obteve as equações do campo gravítico geralmente covariantes - a teoria da relatividade geral (TRG) - e mostrou que a solução esfericamente simétrica destas equações explica a anomalia da precessão da órbita de Mercúrio.

O grande triunfo da TRG teve lugar após a observação do encurvamento dos raios luminosos, durante o eclipse de 29 de Maio de 1919, realizada por Arthur Stanley Eddington (1882-1944), no Príncipe, e Andrew Crommelin, em Sobral, no Brasil. Quando, em Novembro, é anunciado em Londres que estas medidas confirmavam as previsões da TRG, (1) Einstein é aclamado como o génio que destronou Newton. Torna-se de um dia para o outro, aos olhos da opinião pública, no maior e mais famoso cientista de sempre, com a popularidade de uma estrela de cinema, cujas opiniões científicas, políticas ou morais passam a ser escutadas com respeito e admiração. E Einstein, que até

(*) Centro de História das Ciências - Universidade de Lisboa

(* *) Centro de Astronomia e Astrofísica da Universidade de Lisboa

(1) F. W. Dyson, A. Eddington e C. Davidson, "A Determination of the Deflection of Light by the Sun's Gravitational Field”, Royal Society of London. Philosophical Transactions A 220 (1920), 291-333. 
então tinha tido um envolvimento político relativamente discreto, usa a sua celebridade, nos anos que se seguem, na defesa de várias causas que lhe eram caras como o pacifismo, o sionismo, a luta contra o racismo e o desarmamento nuclear. ${ }^{(2)}$

Se a recepção da teoria da relatividade, nas suas versões restrita e geral, tem recebido grande atenção da parte da comunidade internacional de historiadores da ciência, ${ }^{(3)}$ o caso português tem sido pouco estudado no contexto internacional. ${ }^{(4)}$ No plano nacional, este tópico tem recebido atenção principalmente no que concerne à recepção da teoria por parte da comunidade de matemáticos. ${ }^{(5)}$ Propomo-nos aqui contribuir para tornar conhecida uma outra faceta desta história. Com efeito, o impacto da observação do eclipse de 1919 e de outras actividades associadas à apropriação da "nova física" no nosso país, particularmente junto da comunidade astronómica portuguesa, está especialmente associado aos astrónomos do Observatório Astronómico de Lisboa $(\mathrm{OAL})^{(6)}$ e à preparação da expedição britânica à ilha do Príncipe.

\section{Ciência à maneira de Einstein: Teorias de princípio}

Num artigo em que reflecte sobre os métodos da física teórica e em que caracteriza afinal a sua maneira de fazer ciência, Einstein refere-se às chamadas «teorias de princípio», que postulam regras gerais a que todos os fenómenos devem obedecer, por oposição às teorias «construtivas», como a mecânica

(2) A. Einstein, Ideas and Opinions (Nova Iorque: Three Rivers Press, n/d, publicado originalmente em 1954). Ver por exemplo F. Jerome, "Einstein, race, and the myth of the cultural icon," ISIS, 95 (2004), 627-39.

(3) Por exemplo consultar T. Glick, The comparative reception of relativity (Dordrecht: D. Reidel Publishing Company, 1988) e referências aí incluídas.

(4) D. Lopes Gagean, M. Costa Leite, "General relativity and Portugal : a few pointers towards peripheral reception studies” in J. Eisenstaedt, A.J. Kox, orgs., Studies in the History of General Relativity (Boston: Birkhäuser, 1988), pp. 3-14.

(5) A. J. Fitas, “A Teoria da Relatividade em Portugal no Período entre Guerras”, Gazeta de Física, 27 (2004), 4-10.

(6) E. Mota, P. Crawford, A. Simões, "The appropriation of the 'new physics' in a European periphery. The impact of the light bending experimental test in the Portuguese context” in $1^{\circ}$ Congresso Internacional da European Society for the History of Science, subordinado ao tema "Science in Europe-Europe in Science", Maastricht, 4-6 Novembro 2004. 
estatística. ${ }^{(7)} \mathrm{Na}$ sua investigação recorre simultaneamente a experiências de pensamento, às quais aplica seguidamente os chamados «princípios» para depois extrair deles todas as suas consequências lógicas. Tanto nos trabalhos do annus mirabilis de 1905, entre os quais se encontra a formulação da teoria da relatividade restrita (TRR), ${ }^{(8)}$ como em trabalhos subsequentes, Einstein recorre constantemente a este tipo de abordagem, a imagem de marca da sua forma de fazer ciência.

Embora a TRR tenha estendido a relatividade do movimento às leis do electromagnetismo, ela não é compatível com a lei de gravitação de Newton. Segundo Newton, se a distribuição de matéria mudasse numa certa região do espaço, a alteração correspondente do campo gravitacional seria sentida instantaneamente em qualquer outra parte do Universo. Isto não só significava que poderíamos enviar sinais mais rápidos do que a luz, mas exigia também um tempo absoluto, abolido pela teoria da relatividade. Einstein estava consciente deste problema quando, no Outono de 1907, Johannes Stark lhe pediu para contribuir com um artigo de revisão sobre o princípio da relatividade para o Jahrbuch der Radioaktivität und Elektronik. Por essa mesma altura, Einstein começava também a ficar insatisfeito com a limitação da relatividade aos sistemas inerciais e ansiava por estendê-la a todos os observadores.

É então que Einstein, a trabalhar ainda na Repartição de Patentes de Berna, teve «o pensamento mais feliz» da sua vida, como recordou em mais do que uma ocasião. ${ }^{(9)} \mathrm{A}$ igualdade entre massa inercial e massa gravitacional devia ser uma indicação da conexão íntima entre inércia e gravidade. Alguém que se encontre isolado numa caixa fechada não é capaz de distinguir se está em repouso num campo gravítico ou se está acelerado em sentido oposto numa nave espacial no espaço livre. A esta ligação entre movimento acelerado e gravidade, Einstein chamou «princípio da equivalência»(PE). Descreveria mais tarde esse momento admirável de descoberta na sua lição na

(7) A. Einstein, "On the method of theoretical physics", in Ideas and Opinions, op. cit. (2), $270-6$.

(8) J. Stachel, O Annus Mirabilis de Albert Einstein (Lisboa: Gradiva, 2005) contém a tradução dos artigos de 1905.

(9) A. Einstein, "Grundgedanken und Methoden der Relativitätstheorie, in ihrer Entwicklung dargestellt”, in M. Janssen, R. Schulmann et. al., orgs., Collected Papers (Princeton: Princeton University Press, 2001), vol. 7. Artigo escrito para a Nature em 1920 e que nunca foi publicado. 
Universidade de Quioto em 1922. ${ }^{(10)}$ Daí em diante Einstein passou a ligar o problema da gravidade com o problema da extensão da relatividade a todos os observadores. Publicará pela primeira vez as suas reflexões sobre a relação entre o princípio da relatividade e a gravitação no artigo de revisão de 1907, um dos seus trabalhos mais originais. ${ }^{(11)} \mathrm{E}$ só voltará a pensar nestes problemas em Praga, para onde vai em 1911.

\section{Das experiências de pensamento aos testes observacionais}

Einstein não foi o único físico a tentar conciliar uma teoria da gravitação com as ideias da teoria da relatividade. Em Agosto de 1913, fez uma avaliação das outras alternativas e compara-as com a teoria que está a construir, numa carta enviada a Erwin Finlay Freundlich, jovem astrónomo do Observatório de Berlim. Tỉnha chegado à conclusão de que a análise empírica dos poucos testes previstos era a única via para confirmar se a sua intuição estava fisicamente correcta, estando preparado para abandonar a sua teoria, e com ela os princípios em que assentava, se se verificasse uma contradição com as observações. ${ }^{(12)}$ Einstein retomara a construção da teoria da gravitação em Praga, em Abril de 1911, e previra então dois efeitos: o deslocamento gravitacional para o vermelho das riscas espectrais e o encurvamento dos raios luminosos.

Os contactos com Freundlich tinham começado nesse ano em Berlim, por intermédio de Leo W. Pollak, um astrónomo da Universidade de Praga, que revelou a Freundlich o desapontamento de Einstein com os astrónomos por não levarem a sério a sua proposta de medição do encurvamento da luz junto de um campo gravitacional. Freundlich decide corresponder de imediato às solicitações de Einstein e propõe-se fazer medidas da luz rasando o bordo de Júpiter, para verificar se era deflectida pela gravidade do planeta, tal

(10) A. Einstein, "How I created the theory of relativity", traduzido para o inglês por Yoshimasa A. Ono in Physics Today (Agosto 1982), 45-47.

(11) A. Einstein, "Über das Relativitätsprinzip und die aus demselben gezogenen Folgerungen", Jahrbuch der Radioaktivität und Elektronik, 4. (1907), 411-62. Realmente publicado em Janeiro 22, 1908. Correcções no Jahrbuch 5 (1908), 98-99.

(12) K. Hentschel, Einstein Tower. An Intertexture of Dynamic Construction, Relativity Theory, and Astronomy (Stanford: Stanford University Press, 1997). 
como será mais tarde sugerido pelo astrónomo português Melo e Simas. Procurava assim uma confirmação experimental da teoria que não dependesse de um eclipse total do Sol. Este projecto falha mas Einstein escreve a Freundlich agradecendo os seus esforços e lamentando que a Natureza não tenha providenciado um planeta maior do que Júpiter. ${ }^{(13)}$ Freundlich escreve então a William Wallace Campbell (1826-1938) do Observatório Lick solicitando chapas de eclipses anteriores ${ }^{(14)}$. Mas essas fotografias, que não tinham em conta a luminosidade das estrelas de fundo mas sim o Sol, não permitiram tirar nenhuma conclusão.

Sabe-se hoje que, no caso da luz rasando o bordo do Sol, qualquer teoria da gravitação compatível com o PE prevê uma deflexão de 0,875 ”. Mas na presença de um espaço curvo a deflexão é maior $(1,75$ ”), pelo que a teoria de Newton prevê 0,875 ". Um teste permite assim escolher a boa teoria da gravitação e nomeadamente distinguir entre a proposta einsteiniana e outras alternativas. Enquanto o valor 1,75" podia ser medido em 1915, ano em que Einstein publica a sua teoria da relatividade geral ${ }^{(15)}$ e prevê pela primeira vez este valor - o valor correcto para o desvio - não era então tecnicamente possível observar uma deflexão da ordem de 0,02", tal como a TRG previa no caso de Júpiter.

Na carta que Einstein envia a Freundlich, quando já se encontrava em Zurique, em Agosto de 1913, depois de um período de colaboração intensa com Marcel Grossmann, continua confiante: Estou secretamente bastante convencido que os raios luminosos sofrem realmente deflexão. ${ }^{(16)}$ Cerca de três anos depois, caberá a Eddington confirmar a deflexão durante um eclipse solar total.

(13) Carta de Einstein a Freundlich in M. J. Klein, A.J. Kox, et al., orgs., Collected Papers (Princeton: Princeton University Press, 1993), vol. 5, doc. 336.

(14) Freundlich para W.W. Campbell, 25 de Novembro, 1911, Arquivos Mary Lea Shane do Observatório Lick, Santa Cruz. A ideia de escrever a Campbell foi dada por C.D. Perrine, director do Observatório de Estado da Argentina, que foi por sua vez inspirado por Freundlich a levar a cabo uma expedição a um eclipse total do Sol no Brasil em 1912, mal sucedida devido ao mau tempo.

(15) Einstein comunicou a sua TRG à Academia Prussiana das Ciências, em Berlim em 4, 18 e 25 de Novembro. Estes trabalhos foram depois editados na separata Die Grundllage der allgemeinen Relativitätstheorie, in Annalen der Physik, 49 (1916), pp. 769-822.

(16) Carta de Einstein a Freundlich, meados de Agosto de 1913, in M. J. Klein, A.J. Kox, et al., orgs., Collected Papers (Princeton: Princeton University Press, 1993), vol. 5, doc. 468. 


\section{A aventura de um quaker}

A Primeira Guerra Mundial dificultou a troca de informações entre cientistas. Porém, pouco depois de Einstein ter publicado o artigo sobre TRG, o astrónomo holandês Willem de Sitter (1872-1934) obtém uma cópia e enviaa a um jovem brilhante astrofísico inglês, capaz de entender as cogitações e os cálculos aí apresentados pelo físico alemão. Como teórico de raro talento, Eddington rapidamente reconhece a elegância, os fundamentos lógicos e o potencial da nova teoria e aposta na disseminação das ideias de Einstein não só na Grã-Bretanha como nos Estados Unidos da América. ${ }^{(17)}$ Prepara um trabalho intitulado Report on the Relativity Theory of Gravitation, que publica em Londres em 1918. ${ }^{(18)}$

Em Março de 1917, o astrónomo real britânico, Sir Frank Dyson, chamara a atenção para o eclipse total previsto para Maio de 1919, assinalando tratar-se de uma excelente oportunidade para testar a TRG, pois o Sol teria como fundo a constelação das Híadas, muito rica em estrelas brilhantes.

Nesse mesmo ano, o governo britânico aumenta a idade máxima de mobilização para os 35 anos, numa tentativa de resposta ao esforço de guerra. Eddington era um quaker e, tal como Einstein, um pacifista. Então com 34 anos, vê-se na iminência de ser mobilizado a qualquer momento. Sendo um objector de consciência por motivos religiosos, ${ }^{(19)}$ ficaria sujeito à prisão se recusasse alistar-se, situação que representaria um grave problema para os responsáveis do Trinity College, para o Observatório de Greenwich e para a ciência britânica. Ao fim de três entrevistas e após a intervenção de Dyson, testemunhando a importância de Eddington para uma das expedições que se estavam a organizar para observar o eclipse, a isenção requerida por Eddington foi finalmente concedida a 11 de Julho de 1918 e os preparativos das duas expedições britânicas iniciam-se após o armistício de Novembro.

A 8 de Março de 1919, cerca de quatro meses após terminarem as hostilidades, as duas expedições largam do porto de Liverpool a bordo do H.S.M.

(17) A. Warwick, "Through the convex looking glass. A.S. Eddington and the Cambridge reception of Einstein's General Theory of Relativity" in Masters of Theory. Cambridge and the rise of mathematical physics (Chicago: The University of Chicago Press, 2003), pp. 443-500.

(18) A. Eddington, Report on the Relativity Theory of Gravitation (Londres: Fleetway Press Ltd., 1918)

(19) M. Stanley, “'An Expedition to heal the wounds of war'. The 1919 eclipse and Eddington as Quaker adventurer”, ISIS, 94 (2003), 57-89. 
Anselm e dirigem-se à ilha da Madeira. Aí separam-se. A equipa que se dirigia ao nordeste brasileiro, liderada por Crommelin, do Observatório de Greenwich, continua a bordo do Anselm, chegando ao Pará a 23 de Março. A equipa de Eddington permaneceu no Funchal várias semanas a aguardar transporte. A 3 de Abril embarcam finalmente no cargueiro Portugal, da Companhia Nacional de Navegação, em direcção ao Príncipe, situado um grau acima do equador. A 23 de Abril, entram no pequeno porto de S. António.

Nos preparativos para a expedição ao Príncipe, a Royal Society of London estabeleceu contactos prévios com a Sociedade de Geografia de Lisboa (SGL) e com o OAL. A participação do OAL passou muito especificamente pelo controlo de alguns aspectos de ordem logística, como atesta a descoberta recente, no seu espólio documental, da correspondência trocada entre Eddington e os então director e subdirector do observatório, ViceAlmirante Campos Rodrigues e coronel Frederico Oom, respectivamente. ${ }^{(20)}$ No contacto estabelecido, Eddington e a sua equipa tentaram garantir o apoio das autoridades da ilha, auxílio na marcação das viagens de vapor, uma possível presença de intérpretes bem como informações sobre recursos materiais e humanos de que poderiam dispor na sua estada na ilha.

O sucesso da expedição foi, do ponto de vista de Einstein, total. Os valores observados no Príncipe foram 1,6" $\pm 0,3$, e no Sobral 1,98" $\pm 0,12$, intervalos compatíveis com o valor previsto $(1,75 ") .{ }^{(21)}$ Porém, quando a equipa do Príncipe revelou as primeiras chapas fotográficas apanhou um susto - as primeiras 10 não apresentavam nenhuma estrela e, das restantes 6 , duas tinham 5 estrelas cada, o número mínimo necessário para obter um resultado aceitável. Chapas para comparação das posições das estrelas tinham sido tiradas em Oxford alguns meses antes, mostrando o mesmo campo de estrelas, incluindo as Híades e outras estrelas no Touro. Para Eddington, o momento em que mediu a imagem de uma estrela e verificou que a gravidade do Sol tinha encurvado o espaço através do qual a luz tinha viajado, foi o maior momento da sua vida. Imediatamente, envia um telegrama para Inglaterra: Through clouds, hopeful. Eddington. Mais tarde haveria de recordar estes momentos, em termos poéticos que nos abstemos de traduzir:

(20) Arquivo do Observatório Astronómico de Lisboa. Ref. C-240 (1918/1919): Correspondência Eddington/OAL.

(21) A. M. Nunes dos Santos, C. Auretta, Eddington e Einstein (Lisboa: Gradiva, 1992). 
Our shadow-box takes up all our attention. There is a marvelous spectacle above, and, as the photographs afterwards revealed, a wonderful prominence-flame is poised a hundred thousand miles above the surface of the sun. We have no time to snatch a glance at it. We are conscious only of the weird half-light of the landscape and the hush of nature, broken by the calls of the observers, and the beat of the metronome ticking out the 302 seconds of totatily. ${ }^{(22)}$

Os resultados destas observações foram confirmados por Eddington e Dyson na célebre reunião conjunta da Royal Society of London e da Royal Astronomical Society, de 6 de Novembro de 1919, onde físicos e astrónomos tiveram a oportunidade de ouvir as palavras do patriarca J. J. Thomson, que presidiu à sessão:

Este é o resultado mais importante relacionado com a teoria da gravitação desde os tempos de Newton, e é adequado que seja anunciado numa reunião da Sociedade que tão identificada está com ele. Se se mantiver que os raciocínios de Einstein se confirmam - e acabam de sobreviver a dois testes muito severos ligados ao periélio de Mercúrio e ao presente eclipse - então esta teoria é um dos maiores monumentos do pensamento humano. ${ }^{23)}$

Devemos ter presente que as experiências dos eclipses, tal como grande parte da nova ciência, foram realizadas no limite das técnicas disponíveis. Só a partir dos anos 60, com técnicas de tempo de atraso como as de Shapiro ou o recurso a fontes de rádio que podem ser observadas sem esperar por um eclipse do Sol, foi possível reduzir o erro das observações e ficou demonstrado o encurvamento gravitacional da luz para além de qualquer dúvida razoável. Por isso se encontra amiúde na literatura a afirmação de que a lógica e a beleza da teoria de Einstein fizeram tanto para confirmar as observações de 1919 como as observações fizeram para confirmar a teoria. Mas, apesar das

(22) A. Eddington, Space, Time and Gravitation : An Outline of the General Relativity Theory (Nova Iorque : Harper \& Row, reedição 1959, 1a ed. 1920), p.115.

(23) J.J. Thomson, The Observatory, 42 (1919), 389-398. 
controvérsias, os critérios utilizados por Eddington e Dyson foram considerados consistentes em trabalhos recentes. ${ }^{(24)}$

Realizada a expedição, os astrónomos ingleses contactam uma última vez o observatório de Lisboa enviando, em Agosto de 1919, as ampliações em papel registadas no dia do eclipse, ${ }^{(25)}$ em que se destaca a protuberância única observada e que tão discutida foi, na época, nas revistas científicas. Curiosamente nenhuma informação relativa a esta expedição aparece nos jornais ou revistas portugueses no período que se segue, para além de uma notícia breve, a 15 de Novembro no jornal $O$ Século, sobre a reunião conjunta da Royal Society e da Astronomical Society.

\section{Astronomia versus Astrofísica: um novo papel para os eclipses solares}

No último terço do século XIX, os astrónomos expandiram o seu objecto de estudo de tal forma que, para além da posição e do movimento dos corpos celestes, começaram a investigar a natureza física do Sol, planetas, estrelas ou até nebulosas e galáxias. Esta nova preocupação foi em grande parte uma consequência das novas tecnologias observacionais à disposição dos astrónomos. Estas incluíram a utilização de telescópios de acentuada luminosidade e fina resolução - os grandes reflectores -, a fotografia como ferramenta astronómica e, por último, a espectroscopia astronómica. Com o advento da "nova astronomia", também designada astronomia física, astrofísica ou física solar, a fotografia e a espectroscopia passaram a integrar uma lista de técnicas à disposição das equipas de observadores de eclipses, focando-se o estudo do Sol nomeadamente na granulação da sua superfície e na periodicidade do ciclo das suas manchas. ${ }^{(26)}$

(24) Stanley, "Expedition", op. cit. (19); Peter Coles, "Einstein, Eddington and 1919 Eclipse", e-print astro-ph/0102462 (2001). Opiniões contrárias foram expressas anteriormente em J. Earman, C. Glymour, "Relativity and eclipses : the British expeditions of 1919 and their predecessors," Historical Studies in the Physical Sciences, 11 (1980), 49-85 e H. Collins, T. Pinch, The Golem: What everyone should know about science (Cambridge : Cambridge University Press, 1993).

(25) Arquivo do Observatório Astronómico de Lisboa. Ref. C-240 (1918/1919): Correspondência Eddington/OAL.

(26) Robert W. Smith, "Remaking Astronomy : Instruments and Practice in the Nineteenth and Twentieth Centuries" in Mary Jo Nye, ed., The Cambridge History of Science (Cambridge University Press, 2003), vol. 5, pp. 154-173. 
Ao invés, do ponto de vista da astrometria e para os astrónomos tradicionais, nas rotinas anteriores a 1840), as observações de eclipses solares tinham outros objectivos. As medições de maior interesse prendiam-se com os momentos dos contactos do disco lunar com o disco solar, no começo e no final da totalidade. Estas medições permitiam refinar as estimativas das posições relativas da Terra, Lua e Sol e podiam ser usadas em cálculos de mecânica celeste. Absorvidos com os tempos de contacto na totalidade, os astrónomos não conseguiam debruçar-se simultaneamente sobre os fenómenos qualitativos associados às observações da coroa solar, às proeminências e até à análise da composição da atmosfera solar.

A partir do início do século XX, o interesse principal das observações de eclipses solares totais passou a pertencer fundamentalmente ao domínio da astrofísica. Este facto ajuda-nos a perceber a razão pela qual Frederico Oom (1864-1930), promovido a director do Observatório de Lisboa em 1920, nunca se referiu à ocorrência da expedição, dos seus objectivos e finalidades, apesar de ter sido um dos astrónomos portugueses que, entre 1917 e 1920, mais artigos de divulgação científica publicou, em revistas como The Observatory e Astronomische Nachrichten. Aliás, ele próprio foi activo participante na observação de outros eclipses.

De acordo com as novas linhas orientadoras da astronomia e dadas as limitações de equipamento astronómico do OAL, também se percebe a posição de Oom ao opor-se, desde 1905, à organização de expedições para observações de eclipses fora do território nacional. Num artigo escrito para $O$ Instituto, a revista científica ligada à Universidade de Coimbra, defende que as expedições só se justificariam se o grupo de astrónomos tivesse a certeza de obter dados interessantes para a ciência, possuísse instrumentos caros somente úteis para esse fim e tivesse inventado processos novos para estas observações. Acrescenta ainda que os astrónomos não especializados nesta área e com escassos recursos apenas poderiam acompanhar estas expedições quando tendo lugar em território português, onde o dever de colaboração no estudo se impunha. ${ }^{(27)}$

Estas considerações foram continuadas num outro artigo publicado na mesma revista em 1917, onde analisa as circunstâncias do eclipse do Sol de Maio de 1919 e refere que a trajectória da sombra atravessará, entre outros

(27) F. Oom, “O Futuro Eclipse”, O Instituto 52 (1905), 487-490. 
locais, a Ilha do Príncipe. Prevê que esta ilha tem uma forte probabilidade de vir a ser escolhida como estação de observação do fenómeno, convida os astrónomos portugueses a estudarem as condições geográficas e climáticas que lhe estão associadas e apresenta os cálculos que fez para os tempos de contacto, para um ponto central da ilha. ${ }^{(28)}$

\section{Os astrónomos portugueses e a relatividade}

Apesar das recomendações de Oom, o astrónomo Manuel Peres Júnior (1888-1968), na época director do Observatório Campos Rodrigues em Moçambique e mais tarde director do OAL, tentou acompanhar a expedição inglesa, mas sem êxito pois não conseguiu ultrapassar uma série de questões burocráticas. ${ }^{(29)} \mathrm{O}$ mesmo não sucedeu com a expedição ao Sobral, no Brasil, na qual um grupo de astrónomos brasileiros, chefiados por H. Morize (18601930), do Observatório do Rio de Janeiro, acompanhou os ingleses e realizou observações de física solar, tendo, por isso, objectivos distintos dos que motivavam a equipa britânica. ${ }^{(30)} \mathrm{O}$ contraste entre as reacções dos astrónomos brasileiros e portugueses não pode, contudo, ser justificado pelas razões habitualmente referidas na literatura que a partir da ausência de portugueses inferem o seu desinteresse por estas questões.

Goradas que foram as suas expectativas relativamente à participação na expedição, Peres Júnior veio a tornar-se um adepto das ideias relativistas. Em 1922, organiza o $20^{\circ}$ Congresso da Associação da África do Sul para o Progresso da Ciência, em Lourenço Marques, e dirige a secção de Astronomia e Matemática. Na sua comunicação inaugural discute as relações entre astronomia e física e afirma: (..) Como sabem, a principal confirmação dos pontos de vista de Einstein depende de observações astronómicas. (...) Portanto, no caminho do seu desenvolvimento a física chega a uma bifurcação; espera que

(28) F. Oom, "O Eclipse Total do Sol em 29 de Maio de 1919 visível na Ilha do Príncipe", O Instituto, 64 (2) (1917), 97-98.

(29) Arquivo do Observatório Astronómico de Lisboa. Ref. C-463 (1915-1929): Correspondência M. Peres/Oom (OAL).

(30) I. Castro Moreira e A.A.P. Videira, org., Einstein e o Brasil (Rio de Janeiro: Editora URFJ, 1995). 
a astronomia possa dizer qual dos dois caminhos deve seguir. ${ }^{(31)}$ Entre 1922 e 1923, escreve um opúsculo no qual discute a relatividade restrita e geral, segundo se depreende de manuscritos encontrados no Arquivo do OAL, mas, apesar da insistência de Oom, o livro nunca será publicado. Depois de 1930, corresponde-se com o almirante Gago Coutinho (1868-1959) e participa nas primeiras controvérsias envolvendo a teoria da relatividade. Opondo-se a Gago Coutinho, toma o lado dos relativistas em várias sessões da Academia das Ciências onde é apoiado por Santos Lucas. ${ }^{(32)}$

Até aos finais dos anos 20, dois outros astrónomos participam na recepção da teoria da relatividade em Portugal - A. Ramos da Costa (1875-1939) e M. S. Melo e Simas (1870-1934). Ramos da Costa publica, em 1921 e 1923, dois pequenos livros sobre a teoria da relatividade. ${ }^{(33)} \mathrm{Em} 1924$, apresenta num congresso internacional de matemática em Toronto um artigo intitulado L' enseignement des mathématiques doit être orienté pour l'étude de la Relativité. (34)

Melo e Simas, oficial de artilharia e um dos principais astrónomos do OAL, perfilha as ideias da «Nova Física» nos anos 20 e 30. Publica um artigo sobre relatividade nos apêndices do Almanaque do observatório, em Julho de 1922, do qual destacamos as seguintes afirmações:

O nome de um alemão atrai neste momento as nossas simpatias e a admiração ou curiosidade de todo o mundo culto e não culto. (...) é certo ser a Astronomia das ciências que mais tem a utilizar da teoria da Relatividade, fornecendo-lhe, em troca, as melhores das suas confirmações.(...) Um raio de luz muda de direcção nas proximidades dum campo de gravitação. Isto é, a luz, em vez de se propagar em linha recta, descreve curvas dependentes dos campos de

(31) Arquivo do Observatório Astronómico de Lisboa. Ref. C-463 (1915-1929): Correspondência M. Peres/Oom (OAL); M. Peres Júnior, "The role of Astronomy in the development of Science," South African Journal of Science, 19 (Dezembro 1922), 32-41, 41.

(32) Sessão da Classe de Ciências de 1 de Março de 1934, Boletim da Academia de Ciências de Lisboa (1934-35), 64-71; Sessão da Classe de Ciências de 24 de Janeiro de 1935, Boletim da Academia de Ciências de Lisboa (1934-35), 67-68.

(33) A. Ramos da Costa, A Teoria da Relatividade (Lisboa : Biblioteca Nacional, 1921); A. Ramos da Costa, Espaço, Matéria, Tempo ou a Trilogia Einsteiniana (Lisboa : Imprensa Lucas e C.a, 1923).

(34) $\mathrm{O}$ texto desta comunicação não foi encontrado. 
gravitação que atravessa. Esta consequência foi verificada por ocasião do eclipse do Sol de 29 de Maio de 1919, e expedições se preparam para proceder à confirmação durante o próximo eclipse do Sol de Setembro de 1922. ${ }^{(35)}$

Poucos meses depois, promove um ciclo de 13 conferências sobre a teoria da relatividade, que se inicia em Novembro de 1922 na Universidade Livre, em Lisboa, e conta, segundo os jornais da época, com grande afluência de público.

Pelo que sabemos, é o único astrónomo português a tentar acomodar os novos conhecimentos na sua prática científica. Assim, numa comunicação de 1924 à Academia das Ciências de Lisboa, descreve como, a propósito de um pedido feito pela Astronomische Nachrichten, tentou no OAL a observação da ocultação de uma estrela pelo planeta Júpiter, no dia 7 de Maio de 1923. E explica que:

O processo consistiu em determinar a posição relativa dos dois astros alguns minutos antes e depois da ocultação, por forma a que, partindo da posição de um deles, se pudesse calcular a do outro para certo e determinado momento, obtendo-se assim não só uma série de verificações, difíceis de alcançar por outra forma, mas ainda meios para deduzir os efeitos de qualquer influência na própria ocultação, quer provenientes da irradiação luminosa, quer da atmosfera do planeta, quer ainda de não se ter entrado no cálculo com a teoria da relatividade. ${ }^{(36)}$

Mas é levado a concluir que embora os resultados revelem "uma certa tendência no sentido apontado pela teoria da relatividade," os outros efeitos em acção produzem desvios no mesmo sentido, funcionando como obstáculos à verificação da teoria.

Reconhecemos que, tal como noutros países da Europa, também em Portugal a comunidade dos matemáticos desempenhou um papel importante na recepção da teoria, particularmente depois do I Congresso Luso-Espanhol para o Progresso da Ciência, em 1921, onde a teoria da relatividade foi apresentada aos matemáticos portugueses pelo matemático espanhol Plans y

(35) M.S. Melo e Simas, Almanaques de 1924 (OAL, 1922), pp. 47-57.

(36) M.S. Melo e Simas, “Ocultação de uma estrela por Júpiter”, Jornal de Sciências Matemáticas, Físicas e Naturais da Academia de Sciências de Lisboa (1924), 115-122. 
Freire. ${ }^{(37)}$ Esta comunicação veio a inspirar um curso de Física-Matemática totalmente dedicado à teoria da relatividade, ministrado pelo catedrático Santos Lucas em 1922, na Faculdade de Ciências de Lisboa.

Embora os matemáticos tenham participado nas discussões teóricas até os anos 30, a importância da comunidade dos astrónomos na apropriação da relatividade não pode ser ignorada. Estes desempenharam, como se viu, um papel de relevo não só no que se refere à acomodação de novos argumentos mas também em termos das polémicas científicas em que se envolveram. Depois do curso de Santos Lucas, é sintomático que tenha sido um astrónomo e matemático, Manuel dos Reis (1900-1992) quem, em 1930, fez a apresentação mais completa da relatividade restrita e geral, escrita para as suas provas para professor catedrático na Universidade de Coimbra. ${ }^{(38)}$ Intitulado $O$ problema da gravitação universal, o texto descreve a história das teorias da gravitação de Newton a Einstein, e incorpora as novas ideias no contexto da física, contribuindo para facilitar a apropriação da relatividade pela comunidade nascente dos físicos.

\section{Conclusões}

Em Portugal, como noutros países, as reacções à teoria da relatividade variaram da adesão à resistência, qualquer uma destas envolvendo gradações no espaço e no tempo dependentes do perfil das personalidades envolvidas e das comunidades a que estas pertenciam. Sendo a teoria da relatividade uma teoria construída na interface da física, matemática e astronomia não admira que membros destas três culturas científicas reagissem a ela de maneira diferente e se apropriassem dela de modos também variados. Pretendeu-se aqui analisar as primeiras reacções dos astrónomos portugueses, um grupo até agora eclipsado do discurso histórico, perceber o contexto da astronomia portuguesa do início do século XX, as reacções à expedição de Eddington e as respostas à teoria da relatividade, geral ou restrita, propostas na sua sequência. Em suma, dar um contributo preliminar para o entendimento do processo de apropriação da relatividade pelo grupo de astrónomos portugueses.

(37) J.M. Plans y Freire, "Processo histórico del cálculo diferencial absoluto y su importancia actual”, Madrid: Actas do $1^{\circ}$ Congresso Luso- Espanhol para o Progresso das Ciências, 1921.

(38) Manuel dos Reis, O Problema da Gravitação Universal (Coimbra : Imprensa da Universidade, 1930). 
(Página deixada propositadamente em branco) 


\section{Série}

\section{Documentos}

Imprensa da Universidade de Coimbra

Coimbra University Press

2005

- U

C - 